\title{
Use of Item Response Theory to Examine a Cardiovascular Health Knowledge Measure for Adolescents with Elevated Blood Pressure
}

Stephanie L. Fitzpatrick

Maria M. Llabre

Judith R. McCalla

\author{
Patrice G. Saab \\ Randall Penfield \\ Neil Schneiderman
}

University of Miami, Miami FL

The purpose of this study was to assess the psychometric properties of a cardiovascular health knowledge measure for adolescents using item response theory. The measure was developed in the context of a cardiovascular lifestyle intervention for adolescents with elevated blood pressure. Sample consisted of 167 adolescents (mean age = 16.2 years) who completed the Cardiovascular Health Knowledge Assessment (CHKA), a 34-item multiple choice test, at baseline and post-intervention. The CHKA was unidimensional and internal consistency was .65 at pretest and .74 at posttest. Rasch analysis results indicated that at pretest the items targeted adolescents with variable levels of health knowledge. However, based on results at posttest, additional hard items are needed to account for the increase in level of cardiovascular health knowledge at post-intervention. Change in knowledge scores was examined using Rasch analysis. Findings indicated there was significant improvement in health knowledge over time $[t(119)=-10.3, p<$ .0001]. In summary, the CHKA appears to contain items that are good approximations of the construct cardiovascular health knowledge and items that target adolescents with moderate levels of knowledge.

Keywords: Health knowledge, item response theory, classical test theory, confirmatory factor analysis, adolescents

The prevalence rate of cardiovascular disease risk factors among children and adolescents is on the rise. According to NHANES data, 19882002, the prevalence of high blood pressure (i.e., systolic and/or diastolic blood pressure $\geq 95^{\text {th }}$ percentile) among children and adolescents increased from 2.7\% to 3.7\% (Din-Dzietham, Liu, Bielo, \& Shamsa, 2007). During 1999-2008, the percentage of 12-19 year olds with a body mass index $\geq 85^{\text {th }}$ percentile went from $14.8 \%$ to $34.2 \%$ (Ogden et al., 2006; Ogden, Carroll, Curtin, Lamb \& Flegal, 2010). Despite increasing risk for cardiovascular disease (CVD) among youth, the literature indicates that adolescents have a low perception of risk and poor knowledge regarding CVD and prevention (Collins, Dantico, Shearer, \& Mossman, 2004; Vanhecke, Miller, Franklin, Weber, \& McCullough, 2006).

Obtaining sufficient cardiovascular health knowledge plays a role in making appropriate health decisions and initiating behavior change (Homko et al., 2008; Potvin, Richard, \& Edwards, 2000). Although 


\section{FITZPATRICK ET AL.}

cardiovascular lifestyle interventions have been found to increase health knowledge among adolescents (Edmundson et al., 1996; Fardy et al., 2000; Harrell et al., 1998; Miller, King, Miller, \& Kleindorfer, 2007), recent studies suggest that improvement in health knowledge does not necessarily translate into actual health behavior change (Baranowski, Cullen, Nicklas, Thompson, \& Baranowski, 2003; Frost, 1992; Smalley, Wittler, \& Oliverson, 2004). The lack of association between health knowledge and health behavior may be because knowledge is only one component of the process of behavior change. There are other mechanisms of change such as self-efficacy and readiness for change that also need to improve in order for an individual to initiate behavior change. Previous research indicates that health knowledge is related to self-efficacy and readiness for change (Beech, Rice, Myers, Johnson, \& Nicklas, 1999; Gracey, Stanley, Burke, Corti, \& Beilin, 1996) and that self-efficacy mediates the relationship between health knowledge and dietary changes (Rimal, 2000), suggesting that health knowledge has an indirect relationship with health behavior change. Although common theories of behavior change (e.g., Social Cognitive Theory, Theory of Planned Behavior, and Transtheoretical Model) do not explicitly mention knowledge, the role of health knowledge is implicit. Individuals must understand their risks, why they should engage in the healthy behavior, and how to engage in the behavior change (e.g., how to read nutrition labels in order to buy and prepare healthy foods) to revise outcome expectancies, increase perceived behavioral control, and increase readiness for change, processes that are stated in the Theory of Planned Behavior (Ajzen \& Madden, 1986; Fishbein \& Ajzen, 1975) as well as the Transtheoretical Model (Prochaska \& DiClemente, 1984). Therefore, knowledge is an important construct to assess using measures with good psychometric properties.

Recent literature suggests that item response theory (IRT) should be used to develop and improve measurement of constructs often assessed in behavior change interventions (Dunn, Resnicow, \& Klesges, 2006). IRT applies a model-based approach to measurement for each test item that gives the probability of a correct response as a function of the participant's latent trait (e.g., true health knowledge) and the properties of the item (Embretson \& Reise, 2000). IRT may be useful for designing a measure as well as examining the psychometric properties of an existing measure including reliability, construct validity, item difficulty, and item discrimination. In contrast to classical test theory (Hambleton \& Jones, 1993; Wilson, Allen, \& Li, 2006) IRT places item difficulties and person factor scores on the same metric, making it easier to establish which items target the construct of interest (e.g., health knowledge) and are appropriate for the given population. Furthermore, the placement of item difficulties and person factor scores on the same metric has implications 
for assessing change over time. Typically, using a classical test theory approach, improvement in a construct of interest is examined by calculating change scores. However, the problem with this approach is that a one-unit gain may have a different meaning with respect to an actual gain in health knowledge depending on the initial difficulty of the item. More specifically, scores at pretest may be on a different scale than those at posttest making it difficult to compare scores across test administrations. IRT improves upon these problems by simultaneously estimating the knowledge level of each participant as well as the difficulty level for each item and allowing posttest scores to be examined on the same metric as pretest scores. It should be noted that this paper does not propose that IRT is a better statistical method than traditional approaches as all measurement theories stem from the basic concept of classical test theory (i.e., observed score $=$ true score + error), but IRT improves upon tradition by dealing with some of the limitations of classical test theory stated above.

Data for the present paper are based on a study examining the effect of a cardiovascular lifestyle intervention on lowering resting blood pressure among adolescents with elevated blood pressure. A cardiovascular health knowledge measure was developed in the context of the lifestyle intervention to assess adolescents' factual and skill-based knowledge in the areas of blood pressure, nutrition, physical activity, and stress management at pretest and posttest. The purpose of this current study was to examine the factor structure and the psychometric properties of this cardiovascular health knowledge measure using confirmatory factor analysis (CFA) and IRT.

\section{Method}

\section{Participants}

Prospective participants for the cardiovascular lifestyle intervention, age 15-17, were identified during annual high school blood pressure screenings from 2000-2005 in Miami, Florida. Eligibility criteria consisted of having elevated blood pressure (i.e., systolic blood pressure and/or a diastolic blood pressure greater than or equal to the $90^{\text {th }}$ percentile adjusted for sex, age and height), but less than 160/100, and living in the United States for at least four years. Adolescents that had elevated blood pressure at the school screen and follow-up home visit were invited to attend baseline assessments. There were 167 participants randomized into one of three treatment conditions (i.e., minimal, moderate, and intense). Mean age for the sample was 16.2 years, and 
ethnic background consisted of 47\% Hispanic, 35\% Black, 10\% White, and $8 \%$ who did not endorse a racial/ethnic group. Mean parental years of education was 13.26 years, and $64 \%$ of participants endorsed having a family history of high blood pressure. Retention rates at the three-month follow-up were $66 \%$ minimal, $87 \%$ for moderate, and $85 \%$ for the intense condition. For the six-month follow-up there were $64 \%$ retained from the minimal condition, $75 \%$ from moderate, and $87 \%$ from the intense condition.

\section{Procedures}

The study was approved by the University of Miami Institutional Review Board. Parental written informed consent and participant assent was obtained at a home visit during which eligibility for the study was determined. Demographic variables such as age, gender, racial/ethnic background, and parent education were also obtained during the home visit. Procedures for medical and psychosocial assessments have been previously reported (Cugnetto et al., 2008). The current paper will discuss only the collection of health knowledge data.

\section{Cardiovascular Health Knowledge Assessment (CHKA)}

The purpose of the CHKA (see Appendix for items) was to assess adolescents' knowledge of CVD facts and risk factors as well as knowledge of skills needed to make a healthy behavior change. Evidence of content validity was supported by use of a panel of experts to create 34 multiple choice items based on evidenced-based cardiovascular health research as well as content provided in the cardiovascular lifestyle intervention manual and sessions. The panel of experts reviewed the content of each item and decided the items were appropriate for the purpose of the test. Similar to the Know Your Body Questionnaire (Williams, Carter, \& Eng, 1980), the content of the items on CHKA represent several different risk factors for cardiovascular disease including 7 items on blood pressure, 11 items on nutrition, 5 items on physical activity/ exercise, and 11 items on stress management. Participants received the CHKA at baseline and immediately post-intervention. The multiple choice test was scored as correct or incorrect.

\section{Cardiovascular Lifestyle Intervention}

After baseline assessments were completed, participants were randomized into one of three psycho-educational treatments that varied in demand. Using a cognitive behavioral approach, these treatment conditions were designed to promote a healthy lifestyle by improving 
nutrition, increasing physical activity and managing stress. All participants received a lifestyle workbook, which provided general information and recommendations discussed in the sessions and homework sheets, as well as supplemental materials on nutrition, activity, exercise, stress, smoking, and drinking. The minimal condition $(\mathrm{n}=58)$ consisted of a single 90minute session with at least one parent and the adolescent. In the moderate condition $(n=63)$, adolescents attended 10 group sessions over the course of three months. The intense condition $(n=46)$ consisted of 6 individual and 12 group sessions with adolescents over the course of 6 months. Each group session began with participants being introduced to and eating healthy snacks and then jumping rope up to 5 minutes for physical activity. Group sessions consisted of an educational as well as behavioral skill development component that ranged from teaching how to eat healthier diets and increase exercise to practicing relaxation techniques such as diaphragmatic breathing to using role-play for assertiveness and problem solving training.

\section{Statistical Analyses}

Psychometric properties of the CHKA were examined using CFA and IRT. Because the items are intervention specific, discarding items based on psychometric properties at pretest may result in loss of information, power, and thus reliability. We suspected that examining item-level functioning at posttest may also be informative in developing a reliable and valid health knowledge measure for future use as well as determining intervention effects. For instance, we expected that harder items would be easier at posttest if the intervention was effective, while keeping the range in difficulty at an appropriate level for the target group. Furthermore, configural invariance (i.e., items measure the same construct at different times) would be assumed if, indeed, the items are measuring the same construct, but to test this assumption scores from pretest and posttest were needed. Data were available and analyses were conducted on 160 participants at pretest and 127 at posttest. There were no missing data at the item level for both pretest and posttest. Prior to performing the IRT analysis, a CFA was conducted using Mplus version 6.11 to evaluate the factor structure of the CHKA at pretest and posttest. Configural invariance, examining if the pattern of fixed and free parameters or factor structure was the same at pretest and posttest (Horn \& McArdle, 1992) was tested by combining pretest and posttest data into the same model and correlating the error variances for each item across time. Standardized factor loadings calculated in the CFA were used to examine item correlation with the health knowledge factor. We determined goodness of fit of the CFA models to the data by several fit indices including a non- 


\section{FITZPATRICK ET AL.}

significant $\chi^{2}(p>.05)$, comparative fit index (CFI) greater than or equal to .90 , root mean square error approximation (RMSEA) less than or equal to .08 , and the standardized root mean square residual (SRMR) less than .10 (see Kline, 2005 for a discussion on fit indices).

The IRT analysis was conducted using WINSTEPS. There are several models used in IRT, but the Rasch model was applied given the study's sample size and the opportunity to examine item difficulty on the same scale as latent trait ability. In the Rasch model, the dependent variable is the item response (correct or incorrect) and the independent variable is the participant's health knowledge, $\theta$. Because the dependent variable is dichotomous and thus has an expected value that is bounded between 0 and 1, the Rasch model uses a nonlinear function linking the response variable to the independent variable. Information regarding item difficulty and item fit statistics (i.e., infit and outfit) for each item were obtained from the WINSTEPS output. Infit and outfit statistics provide information about the fit of the item to the Rasch model. Items with infit and outfit values between 0.7-1.3 were considered to fit the Rasch model (Adams \& Khoo, 1996).

Treatment effect was assessed two ways with the use of Rasch modeling. In the first approach, the factor scores (i.e., theta) obtained from the Rasch model for pretest were compared to the factor scores from the Rasch model for posttest using paired t-test analysis. In order to conduct this analysis, item difficulties at posttest were fixed to those at pretest to place factor scores on the same scale. Secondly, treatment effect was examined by applying a MIMIC (multiple indicators and multiple causes) model approach. In the MIMIC model, item difficulties at posttest were again fixed to item difficulties at pretest. Treatment condition was dummy coded with two vectors, and the minimal condition was considered the reference group. The health knowledge latent variable was regressed on the two dummy vectors. Effect sizes were calculated using the following formula: $b / \sqrt{ }$ variance.

\section{Results}

Cronbach's alpha for the summated scores obtained from the CHKA was .65 at pretest and .74 at posttest. Results (see Tables 1 and 2) indicated that a single factor model had good approximation of the data at pretest $\left[X^{2}(527)=543.49, p=0.29\right.$, CFI $=0.96$, RMSEA $=0.02$, and WRMR $=0.89]$ and good fit according to 3 out of 4 criteria at posttest $\left[\chi^{2}(527)=569.04, p=0.10, \mathrm{CFI}=0.85, \mathrm{RMSEA}=0.03\right.$, and $\mathrm{WRMR}=$ 0.94]. Configural invariance was evaluated with good model fit $\left[\chi^{2}(2175)\right.$

$=2243.86, p=0.15, \mathrm{CFI}=0.90, \mathrm{RMSEA}=0.01$, and $\mathrm{WRMR}=0.95]$ indicating that the factor structure is similar and items measure the same 
Table 1

Cardiovascular Health Knowledge Assessment Item Fit Indices: Pretest ( $n=160$ )

\begin{tabular}{|c|c|c|c|c|c|c|}
\hline Item & \% Correct & $\beta$ & $\begin{array}{l}\text { Infit } \\
\text { MNSQ }\end{array}$ & $\begin{array}{l}\text { Outfit } \\
\text { MNSQ }\end{array}$ & Measure & $\begin{array}{l}\text { Model } \\
\text { SE }\end{array}$ \\
\hline 1. Definition of blood pressure & $48 \%$ & .59 & .90 & .88 & 0.08 & .17 \\
\hline 2. Symptoms of high blood pressure & $79 \%$ & .27 & 1.03 & 1.06 & -1.46 & .20 \\
\hline 3. Risk for high blood pressure & $63 \%$ & .32 & 1.00 & 1.02 & -0.62 & .17 \\
\hline 4. High blood pressure treatment & $91 \%$ & .50 & .96 & .74 & -2.50 & .28 \\
\hline 5. Food groups & $52 \%$ & .37 & 1.02 & 1.04 & -0.11 & .17 \\
\hline 6. Serving size & $33 \%$ & .15 & 1.06 & 1.06 & 0.74 & .17 \\
\hline 7. Servings a day per food group & $48 \%$ & -.09 & 1.18 & 1.19 & 0.08 & .17 \\
\hline 8. Types of fat & $31 \%$ & .22 & 1.02 & 1.02 & 0.87 & .18 \\
\hline 9. Atherosclerosis & $43 \%$ & .46 & .94 & .91 & 0.28 & .17 \\
\hline 10. Desirable cholesterol level & $64 \%$ & .15 & 1.09 & 1.12 & -0.65 & .17 \\
\hline $\begin{array}{l}\text { 11. Saturated fat impact on cholesterol } \\
\text { levels }\end{array}$ & $83 \%$ & .61 & .86 & .79 & -1.77 & .22 \\
\hline 12. Good minerals for blood pressure & $45 \%$ & .02 & 1.11 & 1.11 & 0.19 & .17 \\
\hline 13. Diet & $51 \%$ & .59 & .88 & .86 & -0.08 & .17 \\
\hline 14. Sodium amount per day & $32 \%$ & .23 & 1.02 & 1.12 & 0.80 & .18 \\
\hline 15. Ingredients on food label & $38 \%$ & .49 & .92 & .91 & 0.50 & .17 \\
\hline 16. Calories per gram & $39 \%$ & .28 & 1.01 & 1.01 & 0.48 & .17 \\
\hline 17. Define stressors & $34 \%$ & .60 & .90 & .88 & 0.71 & .17 \\
\hline 18. Types of stressors & $57 \%$ & .27 & 1.03 & 1.04 & -0.33 & .17 \\
\hline 19. Calories in 1 pound & $21 \%$ & -.08 & 1.13 & 1.43 & 1.39 & .20 \\
\hline 20. Regular exercise benefits & $52 \%$ & .66 & .85 & .81 & -0.11 & .17 \\
\hline 21. Definition of aerobic exercise & $71 \%$ & .82 & .77 & .69 & -0.99 & .18 \\
\hline 22. Exercise duration/frequency & $52 \%$ & .31 & 1.03 & 1.03 & -0.20 & .17 \\
\hline 23. Types of aerobic exercise & $57 \%$ & .48 & .95 & .94 & -0.33 & .17 \\
\hline 24. Target heart rate & $41 \%$ & .27 & 1.00 & .98 & 0.36 & .17 \\
\hline 25. Types of relaxation & $39 \%$ & .20 & 1.03 & 1.04 & 0.48 & .17 \\
\hline 26. Fight or flight & $31 \%$ & .18 & 1.03 & 1.06 & 0.87 & .18 \\
\hline 27. Ways to deal with stress & $33 \%$ & -.00 & 1.10 & 1.19 & 0.77 & .18 \\
\hline 28. Automatic thoughts & $24 \%$ & -.21 & 1.16 & 1.30 & 1.24 & .19 \\
\hline 29. 3 A's of stress mgmt. & $22 \%$ & -.49 & 1.24 & 1.70 & 1.35 & .20 \\
\hline 30. Steps of problem solving & $70 \%$ & .72 & .81 & .76 & -0.96 & .18 \\
\hline 31. Food label & $43 \%$ & .48 & .95 & .93 & 0.28 & .17 \\
\hline 32. Physical responses to stress & $78 \%$ & .65 & .86 & .77 & -1.38 & .20 \\
\hline 33. Stress and thoughts & $41 \%$ & .08 & 1.08 & 1.15 & 0.36 & .17 \\
\hline 34. Relapse triggers & $59 \%$ & .47 & .92 & .90 & -0.44 & .17 \\
\hline
\end{tabular}

Note. Factor loadings $(\beta)$ in bold are significant at $p<.05$ level. 
Table 2

Cardiovascular Health Knowledge Assessment Item Fit Indices: Posttest ( $n=127)$

\begin{tabular}{|c|c|c|c|c|c|c|}
\hline Item & \% Correct & $\beta$ & $\begin{array}{r}\text { Infit } \\
\text { MNSQ }\end{array}$ & $\begin{array}{r}\text { Outfit } \\
\text { MNSQ }\end{array}$ & Measure & $\begin{array}{l}\text { Model } \\
\text { SE }\end{array}$ \\
\hline 1. Definition of blood pressure & $67 \%$ & .47 & .94 & .90 & -0.21 & .20 \\
\hline 2. Symptoms of high blood pressure & $83 \%$ & .47 & .96 & .88 & -1.23 & .25 \\
\hline 3. Risk for high blood pressure & $70 \%$ & .52 & .95 & .88 & -0.37 & .21 \\
\hline 4. High blood pressure treatment & $94 \%$ & .30 & 1.01 & 1.25 & -2.38 & .37 \\
\hline 5. Food groups & $74 \%$ & .51 & .94 & .86 & -0.59 & .21 \\
\hline 6. Serving size & $35 \%$ & .21 & 1.06 & 1.15 & 1.27 & .20 \\
\hline 7. Servings a day per food group & $41 \%$ & -.02 & 1.20 & 1.23 & 0.97 & .19 \\
\hline 8. Types of fat & $48 \%$ & .43 & .98 & .97 & 0.72 & .19 \\
\hline 9. Atherosclerosis & $53 \%$ & .50 & .94 & .92 & 0.43 & .19 \\
\hline 10. Desirable cholesterol level & $69 \%$ & .25 & 1.05 & 1.12 & -0.33 & .20 \\
\hline $\begin{array}{l}\text { 11. Saturated fat impact on cholesterol } \\
\text { levels }\end{array}$ & $89 \%$ & .45 & .94 & .94 & -1.73 & .29 \\
\hline 12. Good minerals for blood pressure & $55 \%$ & .11 & 1.14 & 1.21 & 0.39 & .19 \\
\hline 13. Diet & $71 \%$ & .64 & .87 & .82 & -0.46 & .21 \\
\hline 14. Sodium amount per day & $39 \%$ & .04 & 1.17 & 1.26 & 1.12 & .19 \\
\hline 15. Ingredients on food label & $55 \%$ & .32 & 1.03 & 1.04 & 0.36 & .19 \\
\hline 16. Calories per gram & $30 \%$ & .35 & 1.00 & .95 & 1.51 & .20 \\
\hline 17. Define stressors & $44 \%$ & .65 & .91 & .88 & 0.82 & .19 \\
\hline 18. Types of stressors & $80 \%$ & .35 & 1.03 & 1.06 & -0.94 & .23 \\
\hline 19. Calories in 1 pound & $35 \%$ & .30 & 1.05 & 1.07 & 1.31 & .20 \\
\hline 20. Regular exercise benefits & $55 \%$ & .58 & .89 & .89 & 0.36 & .19 \\
\hline 21. Definition of aerobic exercise & $84 \%$ & .72 & .86 & .75 & -1.29 & .25 \\
\hline 22. Exercise duration/frequency & $65 \%$ & .53 & .94 & .92 & -0.13 & .20 \\
\hline 23. Types of aerobic exercise & $80 \%$ & .51 & .95 & .88 & -0.99 & .23 \\
\hline 24. Target heart rate & $52 \%$ & .25 & 1.07 & 1.13 & 0.54 & .19 \\
\hline 25. Types of relaxation & $64 \%$ & .44 & .97 & .96 & -0.05 & .20 \\
\hline 26. Fight or flight & $36 \%$ & .34 & 1.02 & 1.01 & 1.27 & .20 \\
\hline 27. Ways to deal with stress & $41 \%$ & .45 & .96 & .95 & 1.01 & .19 \\
\hline 28. Automatic thoughts & $30 \%$ & .13 & 1.08 & 1.31 & 1.51 & .20 \\
\hline 29. 3 A's of stress mgmt. & $60 \%$ & .29 & 1.03 & 1.01 & 0.14 & .19 \\
\hline 30. Steps of problem solving & $79 \%$ & .55 & .95 & .81 & -0.94 & .23 \\
\hline 31. Food label & $60 \%$ & .31 & 1.03 & 1.01 & 0.10 & .19 \\
\hline 32. Physical responses to stress & $88 \%$ & .63 & .87 & .80 & -1.65 & .28 \\
\hline 33. Stress and thoughts & $61 \%$ & .29 & 1.04 & 1.03 & 0.06 & .19 \\
\hline 34. Relapse triggers & $74 \%$ & .38 & .99 & .91 & -0.59 & .21 \\
\hline
\end{tabular}

Note. Factor loadings $(\beta)$ in bold are significant at $p<.05$ level. 
construct at pretest and posttest. Standardized factor loadings for each item at pretest and posttest can be seen in Tables 1 and 2. There were 18 items at pretest and 24 items at posttest that had significant standardized loadings $\geq .30$ (Kline, 2005; Nunnally \& Berstein, 1994), suggesting that these items are strongly related to the underlying construct (i.e., cardiovascular health knowledge).

\section{Rasch modeling}

Tables 1 and 2 present the weighted fit indices (infit and outfit statistics) and item difficulty (measure) for each item. Overall, items at both pretest and posttest had acceptable infit and outfit values. Item difficulties were based on the parameters calculated in the Rasch model analysis. The metric at both pretest and posttest was set with a mean item difficulty of zero. Figure 1 and Figure 2 present the construct maps for the CHKA at pretest and posttest. Each '\#' is equal to 2 people and each '.' is equal to 1 person. Item difficulties and participant latent trait ability (i.e., cardiovascular health knowledge) are on the same metric using a logit scale, which is centered at a mean of zero. Items located at zero are considered moderately difficult compared to other items on this measure and adolescents at this level have a moderate level of health knowledge compared to other adolescents in the sample. Harder items and adolescents with higher levels of health knowledge are located above zero (positive logits). Easier items and adolescents with lower levels of health knowledge are located below zero (negative logits). At pretest, the item difficulties mapped moderately well onto the distribution of the participant's latent trait ability. These findings suggest that the items are targeting adolescents with variable levels of health knowledge. On the other hand, the construct map at posttest is misaligned, and additional hard items are needed to better target the adolescents' level of cardiovascular health knowledge at post-intervention.

Some items at pretest that were considered hard became easier at posttest after adolescents completed the intervention (see Tables 1 and 2). It should be noted that the item difficulties are similar across pretest and posttest (reflecting the same general rank ordering of the items in terms of difficulty at pretest and posttest) but not identical. This may be due to parameter estimation error as well as shifts in item difficulties due to intervention effects on learning occurring between pretest and posttest that impacted item difficulty differentially. In other words, it is expected that items with content well-aligned with the cardiovascular lifestyle intervention would have a relatively large decrease in difficulty at posttest. There were 16 items at pretest that were considered easier at posttest. 


\section{FITZPATRICK ET AL.}

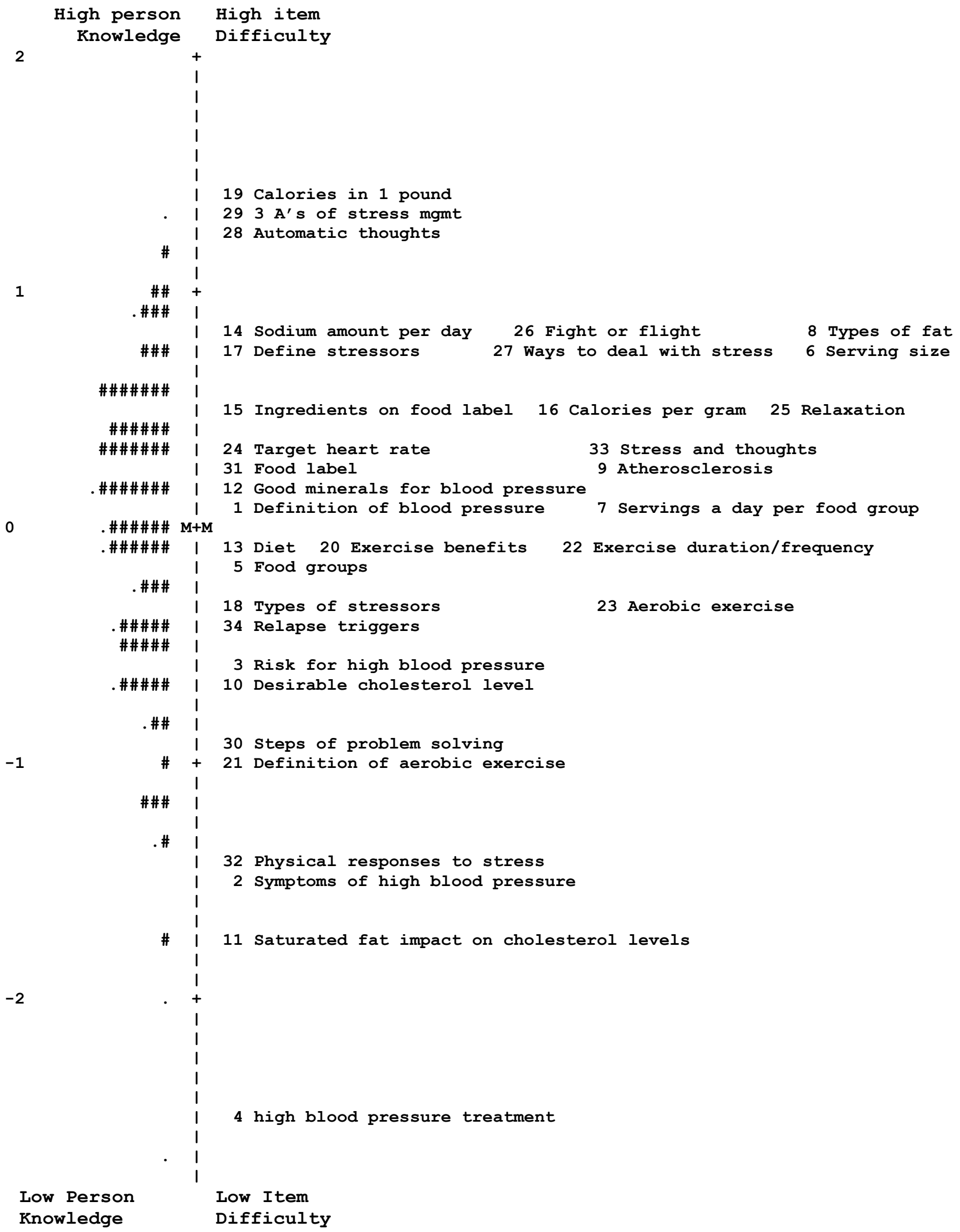

Figure 1. Construct map for cardiovascular health knowledge items at pretest 


\section{EXAMINATION OF A CARDIOVASCULAR HEALTH KNOWLEDGE MEASURE}

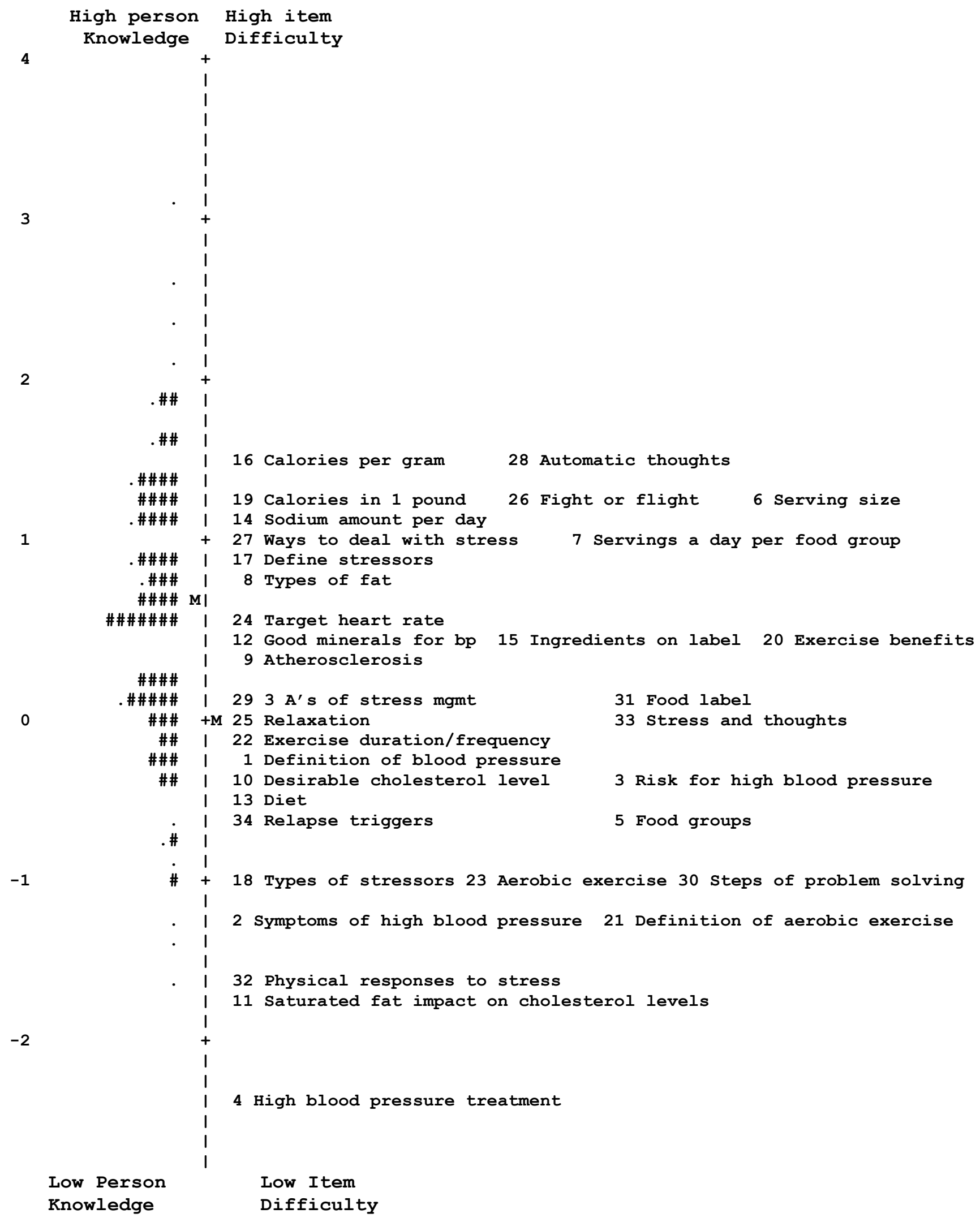

Figure 2. Construct map for cardiovascular health knowledge items at posttest 


\section{Retainment of items and examining gain scores}

Based on results from the CFA, item loadings were compared across pretest and posttest to determine which items should be removed or retained to proceed with the treatment effect analysis. Items that maintained a significant standardized factor loading $\geq .30$ from pretest to posttest were retained. Items that had an increase in standardized factor loading $\geq .30$ and the loading was significant at posttest were retained. Items $4,6,7,10,12,14,24$, and 28 were removed because they did not meet one of the above criteria (see Tables 1 and 2). For the remaining 26 items, Cronbach's alpha was .67 and .77 for pretest and posttest, respectively. Model fit at pretest with the 26 items was $\left[\chi^{2}(299)=322.09\right.$, $p=0.17, \mathrm{CFI}=0.95, \mathrm{RMSEA}=0.02$, and $\mathrm{WRMR}=0.88]$ and at posttest was $\left[\chi^{2}(299)=333.41, p=0.08, \mathrm{CFI}=0.89, \mathrm{RMSEA}=0.03\right.$, and WRMR $=0.93]$ suggesting that removal of the poor indicators of health knowledge slightly improved reliability and construct validity for the CHKA items.

Analysis to examine gain scores or improvement in cardiovascular health knowledge was conducted with the remaining 26 items. Item difficulties at posttest were fixed to difficulty values at pretest. Latent trait ability (i.e., theta) for each participant was obtained at pretest and posttest using Rasch analysis. A paired $t$-test was performed to compare the mean of the thetas at pretest to those at posttest. There was a significant difference in average theta $[t(119)=-10.3, p<.0001]$ at pretest to posttest, suggesting that there was an improvement in cardiovascular health knowledge over time. Furthermore, there were no significant differences between treatment conditions on the cardiovascular health knowledge latent variable at pretest. When item difficulties at posttest were fixed to those at pretest to examine item gains, there were treatment differences. At posttest, the intense condition had a significantly higher level of cardiovascular health knowledge compared to the minimal $(\beta=.50, S E=$ $.12, p<.001 ; d=1.12)$ and moderate conditions $(\beta=.26, S E=.11, p<.05$; $d=.59$ ). Furthermore, the moderate condition had a significantly higher level of health knowledge than the minimal condition $(\beta=.23, S E=.11, p$ $<.05 ; d=.53$ ). These findings suggest that the intervention, specifically the moderate and intense treatment conditions, were effective in improving adolescents' cardiovascular health knowledge.

\section{Discussion}

The study illustrated the application of two approaches: CFA and IRT, to the evaluation of a knowledge measure specifically designed to assess the effect of an intervention. The internal consistency for the CHKA (Cronbach's alpha of .65 at pretest and .74 at posttest) is comparable to 
reliability for cardiovascular health and dietary knowledge measures in other intervention studies (Edmundson et al., 1996; Williams et al., 1980). In addition to obtaining internal consistency for the measure, using CFA and IRT frameworks provided information about the factor structure, difficulty, and fit of the items in order to better determine whether the set of items properly measured the construct of cardiovascular health knowledge. CFA results supported a single factor model at pretest and posttest and configural invariance suggested that the items measured the same construct at pretest and posttest.

Based on the factor loadings from the CFA, there were a number of items at pretest and posttest that had poor item discriminations (i.e., items were not consistently measuring the same construct as the other items). Items that consistently had poor item discrimination from pre to posttest included 7, 12, 14, and 28. The four items asked the following: item $7-$ "Which of the following does not indicate the correct number of servings a day that you should eat?"; item 12 -"What minerals have a positive effect on blood pressure?"; item 14 - "How much sodium is recommended per day?"; and item 28 - "Which is not a characteristic of automatic thoughts?" These items were perhaps measuring other factors in addition to health knowledge. For instance, since the answer for item 14 was provided in milligrams in the intervention manual (i.e., the amount of sodium recommended per day), this item assessed math skills in addition to knowledge because in order to choose the right answer, the participant had to be able to convert milligrams into grams. For items 7,12 , and 28 , it is not as obvious why these items had poor discriminations. Perhaps these items were assessing vocabulary skills in addition to health knowledge. For instance, participants had to be familiar with the word 'servings' to answer 7, 'minerals' for 12, as well as 'automatic thoughts' for 28. Although there were group sessions and written materials provided to define serving size and automatic thoughts, perhaps these were concepts that needed further explanation and/or repetition to make sure the participants understood these terms as well as how to implement them in their daily life.

According to the construct maps, at pretest items mapped moderately well onto person latent trait ability (i.e., health knowledge person factor score) with the exception of one very easy item (item 4 - "Which of the following is not suggested to help high blood pressure?") and two difficult items (item 19 - "How many extra calories must you consume to gain one pound?" and item 29 - "Which is not one of the three A's of stress management?"), suggesting that the remaining items had good coverage of the health knowledge construct. At posttest, there was a clear improvement in health knowledge scores, but a need for additional harder items. Perhaps parallel forms could have been used, parallel meaning that 
items from both forms are significant indicators of the cardiovascular health knowledge construct, but the posttest form would have more items that assessed critical thinking skills (i.e., ability to apply newly gained knowledge) to create more of a challenge. Difficulty among the items varied and in some cases decreased from pretest to posttest. Items that were consistently easy, such as item 4 , suggested the answer for this item (i.e., increase in salt consumption) was not hard to distinguish from the other options (i.e., regular exercise, medications, or stress reduction) and/or this item was not contributing a large amount of information about the health knowledge of the participants. The difficulty level of items 19 and 29 was decreased because the answers to these items were discussed in group sessions and intervention written materials.

There were eight items removed due to having poor item discriminations at either or both pretest and posttest. From the 26 retained items, 16 items had a decrease in item difficulty from pretest to posttest. This change in item difficulty as well as the lack of a consistent scale on which to compare summated pretest and posttest scores could bias results when assessing change in knowledge over time. To avoid this possible issue, using Rasch modeling, participants' latent trait ability or theta at posttest was obtained by fixing the item difficulties at posttest to those at pretest. Results indicated that there was a significant improvement in cardiovascular health knowledge from pretest to posttest, suggesting that the CHKA was sensitive to change. There were no significant differences in level of health knowledge among the treatment conditions at pretest, which suggested that randomization was successful. Given the same level of item difficulty at pretest, findings at posttest suggested adolescents in the intense condition had a significantly higher level of cardiovascular health knowledge than adolescents in the minimal and moderate conditions. Also, the moderate condition had a significantly higher level of cardiovascular health knowledge than the minimal.

Limitations. Psychometric analyses of the CHKA were limited to applying a Rasch model because of the small sample size. The Rasch model only estimates item difficulty and assumes that each item has the same discrimination (i.e., equal to 1). Furthermore, the Rasch model does not account for guessing, which is likely to occur in a multiple choice test. However, by using CFA, item discrimination was assessed and construct validity examined. In contrast, the current study adds to the literature on the utility of examining the psychometric properties of a new measure using both CFA and IRT (Masse, Heesch, Eason, \& Wilson, 2006). Information obtained from the CFA and IRT analysis provides a whole picture about the measure and functioning of the items (Llabre \& Fitzpatrick, 2012). Moreover, CFA helps to satisfy one of the assumptions of IRT regarding appropriate dimensionality for the IRT model, by 
confirming the factor structure of the measure (Embretson \& Reise, 2000).

In summary, by using CFA and Rasch analysis, the construct validity, configural invariance, and item difficulty of the CHKA were examined to determine the appropriateness of these items for a sample of adolescents with elevated blood pressure. The CHKA appears to contain items that are good approximations of the construct, cardiovascular health knowledge, and items that target adolescents with moderate levels of knowledge. Given the intervention specific nature of some of the items, it is not suggested that the CHKA as a whole can be used across studies. However, there are some items that assess aspects of cardiovascular health knowledge (e.g., risk factors and preventive measures for cardiovascular disease) that can be used in other studies with adolescents. This paper has demonstrated the usefulness of using an IRT framework to examine psychometric properties of a measure at the item level to obtain more reliable and valid scores.

Author Note: This work was supported by grant T32HL07426 from the National Institute of Health and NHLBI NIH Program Project 5 P01 HL36588. Correspondence concerning this article should be addressed to Stephanie L. Fitzpatrick, Welch Center for Prevention, Epidemiology, \& Clinical Research, Division of General Internal Medicine, Johns Hopkins School of Medicine, 2024 E. Monument Street, Suite 1-500K, Baltimore, MD 21287, or by email to sfitzpa8@jhmi.edu.

\section{References}

Adams, R. J. \& Khoo, S. (1996). Quest. Melbourne, Australia: ACER.

Ajzen, I. \& Madden, T. J. (1986). Prediction of goal-directed behavior: Attitudes, intentions, and perceived behavioral control. Journal of Experimental Social Psychology, 22, 453-474.

Baranowski, T., Cullen, K. W., Nicklas, T., Thompson, D., \& Baranowski, J. (2003). Are current health behavioral change models helpful in guiding prevention of weight gain efforts? Obesity Research, 11, 23S - 43S.

Beech, B. M., Rice, R., Myers, L., Johnson, C., \& Nicklas, T. A. (1999). Knowledge, attitudes, and practices related to fruit and vegetable, consumption of high school students. Journal of Adolescent Health, 24, 244250.

Collins, K. M., Dantico, M., Shearer, N. B. C., \& Mossman, K. L. (2004). Heart disease awareness among college students. Journal of Community Health, 29, 405-420. 


\section{FITZPATRICK ET AL.}

Cugnetto, M. L., Saab, P. G., Llabre, M. M., Goldberg R., McCalla, J. R., \& Schneiderman, N. (2008). Lifestyle factors, body mass index, and lipid profile in adolescents. Journal of Pediatric Psychology, 33, 761-771.

Din-Dzietham, R., Liu, Y., Bielo, M. V., \& Shamsa, F. (2007). High blood pressure trends in children and adolescents in national surveys, 1963 to 2002, Circulation, 116, 1488-1496.

Dunn, A. L., Resnicow, K., \& Klesge, L. M. (2006). Improving measurement methods for behavior change interventions: opportunities for innovation. Health Education Research, 21, i121-i124.

Edmundson, E., Parcel, G. S., Feldman, H. A., Elder, J., Perry, C. L., Johnson, C. C., Williston, B. J., ... Webber, L. (1996). The effects of the Child and Adolescent Trial for Cardiovascular Health upon psychosocial determinants of diet and physical activity behavior. Preventive Medicine, 25, 442-454.

Embretson, S. E. \& Reise, S. P. (2000). Item Response Theory for Psychologists. Mahwah, New Jersey: Lawrence Erlbaum Associates.

Fardy, P. S., Azzollini, A., Magel, J. R., White, R. E. C., Schmitz, M. K., Agin, D., Clark, L. T., ... Tekverk, L. (2000). Gender and ethnic differences in health behaviors and risk factors for coronary disease among urban teenagers: The PATH Program. The Journal of Gender-Specific Medicine, 3, 59-68.

Fishbein M. \& Ajzen, I. (1975). Belief, Attitude, Intention and Behavior: An Introduction to Theory and Research. Reading, MA: Addison-Wesley.

Frost, R. (1992). Cardiovascular risk modification in the college student: Knowledge, attitudes, and behaviors. Journal of General Internal Medicine, 7, 317-320.

Gracey, D., Stanley, N., Burke, V., Corti, B., \& Beilin, L. J. (1996). Nutritional knowledge, beliefs, and behaviours in teenage school students. Health Education Research: Theory \& Practice, 11, 187-204.

Hambleton, R. K. \& Jones, R. W. (1993). Comparison of classical test theory and item response theory and their applications to test development. Educational Measurement: Issues and Practices, 12, 38-47.

Harrell, J. S., Gansky, S. A., McMurray, R. G., Bangdiwala, S. I., Frauman, A. C., \& Bradley, C. B. (1998). School-based interventions improve heart health in children with multiple cardiovascular disease risk factors. Pediatrics, 102, 371-380.

Homko, C. J., Santamore, W. P., Zamora, L., Shirk, G., Gaughan, J., Cross, R., Kashem, A., ... Bove, A. A. (2008). Cardiovascular disease knowledge and risk perception among underserved individuals at increased risk of cardiovascular disease. Journal of Cardiovascular Nursing, 23, 332-337.

Horn, J. L. \& McArdle, J. J. (1992). A practical and theoretical guide to measurement invariance in aging research. Experimental Aging Research, $18,117-144$.

Kline, R. B. (2005). Principles and Practice of Structural Equation Modeling, Second Edition. New York, NY: The Guilford Press.

Llabre, M. M. \& Fitzpatrick, S. L. (2012). Revisiting measurement models in psychosomatic medicine research: A latent variable approach. Psychosomatic Medicine, 74, 169-177. 
Miller, E. T., King, K. A., \& Kleindorfer, D. (2007). FAST Stroke Prevention Educational Program for middle school students: Pilot study results. Journal of Neuroscience Nursing, 39, 236-242.

Nunnally, J. C., Bernstein, I. H. (1994). Psychometric Theory, Third Edition. New York, NY: McGraw-Hill.

Ogden C. L., Carroll M. D., Curtin L. R., McDowell M. A., Tabak C. J.,\& Flegal K. M. (2006). Prevalence of overweight and obesity in the United States, 19992004. JAMA, 295, 1549-1555.

Ogden C. L., Carroll M. D., Curtin L. R., Lamb M. M., \& Flegal K. M. (2010). Prevalence of high body mass index in US children and adolescents, 20072008. JAMA, 303, 242-249.

Potvin, L., Richard, L., \& Edwards, A. C. (2000). Knowledge of cardiovascular disease risk factors among the Canadian population: relationships with indicators of socioeconomic status. Canadian Medical Association Journal, $162, \mathrm{~S}_{5}-\mathrm{S} 11$.

Prochaska, J. O. \& DiClemente, C. C. (1984). The Transtheoretical Approach: Crossing Traditional Boundaries of Change. Homewood, IL: Dow Jones/Irwin.

Rimal, R. N. (2000). Closing the knowledge-behavior gap in health promotion: The mediating role of self-efficacy. Health Communication, 12, 219-237.

Smalley, S. E., Wittler, R. R., \& Oliverson, R. H. (2004). Adolescent assessment of cardiovascular heart disease risk factor attitudes and habits. Journal of Adolescent Health, 35, 374-379.

Vanhecke, T. E., Miller, W. M., Franklin, B. A., Weber, J. E., \& McCullough, P. A. (2006). Awareness, knowledge, and perception of heart disease among adolescents. European Journal of Cardiovascular Prevention and Rehabilitation, 13, 718-723.

Williams, C. L., Carter, B. J., \& Eng, A. (1980). The "Know Your Body" program: A developmental approach to health education and disease prevention. Preventive Medicine, 9, 371-383.

Wilson, M., Allen, D. D., \& Li, J. C. (2006). Improving measurement in health education and health behavior research using item response modeling: comparison with the classical test theory approach. Health Education Research, 21, i19-i32. 


\section{FITZPATRICK ET AL.}

\section{Appendix \\ CARDIOVASCULAR HEALTH KNOWLEDGE ASSESSMENT \\ Please answer the following: (Correct answers are in bold.)}

1. What is blood pressure?

a) the amount of blood the heart pumps out with each beat

b) how fast the heart beats

c) the force of blood against the walls of the arteries of the body

d) how long it takes the blood to circulate through the body

2. What does high blood pressure feel like?
a) a tightness in the chest
b) like you have just run up a flight of stairs
c) a dull throbbing pain on the right side of your chest
d) generally, there are no symptoms

3. Which of the following people would be LEAST likely to have high blood pressure?
a) a white woman who teaches step aerobics
b) a black man who teaches step aerobics
c) a white woman who eats a diet high in salt
d) a black man who eats a high diet in salt

4. Which of the following is NOT suggested to help high blood pressure?
a) regular exercise
b) medications
c) stress reduction
d) increase in salt consumption

5. From top to bottom, what are the groups in the food pyramid?
a) dairy, fats and oils, grains, meat, vegetables and fruits
b) fats, oils and sweets, meat, dairy, grains, vegetables and fruits
c) grains/breads, dairy, vegetables and fruits, fats, oils and sweets
d) fats, oils and sweets, dairy, meat, vegetables and fruits, grains

6. All of the following are examples of a serving EXCEPT:
a) an 8 oz. glass of milk
b) a bagel
c) a medium apple
d) a medium hamburger patty

7. Which of the following does NOT indicate the correct number of servings a day that you should eat?
a) dairy: 2-4 servings
b) grain/bread: 4-5 servings
c) fruit: 2-4 servings
d) vegetables: 3-5 servings 
8. Which type of fat is solid at room temperature?
a) saturated or hydrogenated fat
b) polyunsaturated fat
c) monosaturated fat
d) all of the above

9. What is atherosclerosis?
a) when the heart cannot pump enough blood to the body with each heart beat
b) when the chambers in the heart are too small
c) an irregular heart beat
d) when the inside of the blood vessels become coated with fat

10. What is the desirable level of cholesterol to maintain for good health?
a) less than 100
b) less than 200
c) less than 300
d) less than 400

11. What does eating a large amount of saturated fat do to cholesterol levels?
a) raise them
b) lower them
c) keeps them safe
d) it depends on the particular type of food eaten

12. What minerals have a positive effect on blood pressure?
a) calcium and zinc
b) zinc and magnesium
c) calcium and potassium
d) potassium and magnesium

13. What substances should you decrease in your diet?
a) caffeine, fiber
b) sodium, caffeine
c) fiber, sodium
d) all of the above

14. How much sodium is recommended per day?
a) Less than 2.4 grams
b) Less than 24 grams
c) Less than 240 grams
d) there is no recommended amount 
15. How are ingredients on the food label listed?
a) in order of amount in a product, with the ingredient in largest quantity first, down to the smallest quantity
b) in order of amount in a product, with the ingredient in smallest quantity first, up to the largest quantity
c) alphabetically
d) it depends on the brand of the food

16. What is the correct number of calories per gram of the following substances?
a) fats $=4$, protein $=9$, carbohydrates $=4$
b) fats $=4$, protein $=4$, carbohydrates $=9$
c) fats $=9$, protein $=4$, carbohydrates $=4$
d) fats $=9$, protein $=9$, carbohydrates $=4$

17. What are stressors?
a) the physical responses that occur when a person is under stress
b) situations and thoughts that cause stress
c) the emotional responses that occur when a person is under stress
d) all of the above

18. Which of the following are NOT stressful?
a) taking an important exam
b) a new romance
c) the death of a relative
d) none of the above

19. How many extra calories must you consume to gain one pound?
a) 1000
b) 2500
c) $\mathbf{3 5 0 0}$
d) 4000

20. Which of the following does NOT result from regular exercise?
a) decreases in muscle tension
b) increase of oxygen to the brain
c) increase in circulation
d) increase in blood pressure

21. What is aerobic exercise?
a) exercise that conditions the heart and lungs
b) exercise for the purpose of increasing muscle mass
c) exercise that makes the kidneys more efficient
d) exercise that is done to music 
22. How often should you do aerobic exercise to get its full benefits?
a) at least 5 times a week for a minimum of 10 minutes
b) at least 5 times a week for a minimum of 20 minutes
c) at least 3 times a week for a minimum of 10 minutes
d) at least 3 times a week for a minimum of 20 minutes

23. Which of the following is an example of aerobic exercise?
a) gardening
b) weight lifting
c) yoga
d) swimming

24. What should your target heart rate be during aerobic activity?
a) $25-50 \%$ of your maximum heart rate
b) $50-65 \%$ of your maximum heart rate
c) $70-85 \%$ of your maximum heart rate
d) $85-100 \%$ of your maximum heart rate

25. All of the following are forms of relaxation except?
a) Deep Breathing
b) PMR
c) REM
d) All of the above

26. What do you call the physical reaction a person's body has that prepares him/her to meet a challenging situation?
a) alertness
b) fight or flight response
c) vigilance
d) self preservation response

27. Good ways to deal with a stressful situation include all of the following EXCEPT:
a) practice relaxation
b) rehearse for it
c) expect some stress
d) try not to think about it

28. Which is NOT a characteristic of "automatic thoughts?"
a) they are often irrational
b) they are hard to turn off
c) they are positive self-statements
d) they are learned 
29. Which is NOT one of the three A's of stress management?
a) Avoid
b) Assess
c) Alter
d) Adapt

30. Problem-solving consists of all of the following steps EXCEPT:

a) considering the consequences

b) avoiding the problem

c) evaluating the outcome

d) making a decision

31. What information is NOT provided on a typical food label?
a) number of calories per serving
b) grams of fat
c) number of servings required per day
d) percentage of your daily requirement of certain vitamins and minerals

32. Physical responses to stress include all of the following EXCEPT:
a) voice deepening
b) increased blood pressure
c) increased heart rate
d) muscle tension

33. Which is the most important thing that influences how you feel in a stressful situation?
a) what you think about the situation
b) what the situation actually is
c) who is with you time at the time
d) your body's physical condition

34. What usually triggers a relapse?
a) something very good happening to you
b) forgetfulness
c) negative emotions or stressful events
d) nobody knows what triggers a relapse 